\title{
Medicamentos esenciales, patentes y licencias obligatorias: Doha no es la respuesta
}

\author{
Salvador Bergel \\ Universidad de Buenos Aires \\ s.bergel@ezabog.com.ar \\ María Julia Bertomeu \\ Consejo Nacional de Investigaciones Científicas y Técnicas (CIF-INEO) \\ mjbertomeu@gmail.com
}

Fecha de recepción: 26-6-2020

Fecha de aceptación: 28-6-2020

\section{Resumen}

Es frecuente buscar en la Declaración de Doha (Organización Mundial del Comercio, 2001) una base para la protección de la salud pública mundial confiando en las licencias obligatorias (compulsory license). Pero no cabe llamarse a engaño, pues se trata de un acuerdo impulsado por la así llamada BigPharma en el mismo momento en que arrasaba con los derechos de los países en vías de desarrollo, que no aceptaban las patentes sobre productos farmacéuticos. El objetivo de nuestro trabajo es mostrar que las patentes de invención en el campo farmacéutico consagran derechos patrimoniales claramente incompatibles con el derecho universal a la salud y en cierta medida también a la vida. Es absurdo hacer competir el derecho humano a la salud con los derechos patrimoniales de protección de creaciones intelectuales, incluso reconociendo las enmiendas de Doha.

Palabras clave: acuerdo ADPIC; acceso a los medicamentos; salud pública

\section{Abstract. Essential drugs, patents and compulsory licenses: Doha is not the answer}

The Doha Declaration (World Trade Organization, 2001) is often used as the basis for arguments to protect global public health, and to trust that compulsory licenses can do so. But one should not be deceived about this proposal. This is an agreement promoted by the so-called Big Pharma at the very same time it was violating the rights of developing countries that refused to enforce patents of pharmaceutical products. The purpose of this paper is to show that pharmaceutical patents produce proprietary rights that are clearly incompatible with the universal right to health, and to a certain extent the right to life. For this reason, it is absurd to claim that there is a competition between the human right to health with this supposed proprietary right to the protection of intellectual property, even if one accepts the Doha amendments.

Keywords: TRIPS Agreement; access to medicines; public health 


\section{Sumario}

\section{Introducción}

2. Sobre el acceso a los medicamentos esenciales y las patentes de invención

3. Doha y las licencias obligatorias
4. A modo de conclusión

Referencias bibliográficas

\section{Introducción}

Ante la probabilidad de que finalmente se logre una vacuna para combatir la COVID-19, la prensa nacional e internacional no cesa de hablar sobre su producción y distribución y, muy especialmente, sobre la posibilidad de que los países ricos acaparen sus existencias. Todo ello nos trae a la memoria un viejo e inacabado debate sobre los derechos de propiedad intelectual que se ejercen sobre los productos farmacéuticos ${ }^{1}$.

Es indiscutible que el derecho al acceso a los medicamentos esenciales forma parte de un derecho más general a la atención de la salud, aunque urge recordar que tal derecho está íntimamente relacionado con el sistema de patentes de invención que, a nivel internacional, nació con el Convenio de París sobre la propiedad industrial del año 1883, cuando se concedió un importante margen de libertad sobre la duración y la explotación de los derechos de invención y especialmente sobre los procedimientos, aunque no así sobre los productos farmacéuticos ${ }^{2}$. A medida que la industria farmacéutica reforzó su presencia y aumentó de manera descomunal sus ganancias en el mercado (Baker, 2018) ${ }^{3}$, comenzó a presionar fuertemente para obtener una protección mayor por la vía de una legislación acorde con sus demandas. Este proceso tiene una historia que relataremos de manera breve.

1. El grueso de las publicaciones que recogen los debates apenas van más allá del año 2008, de ahí que hablemos de una vieja discusión, aunque inacabada. Nuestro objetivo es contribuir a reabrir ese debate en un punto muy específico, argumentando a favor de una posición que no es mayoritaria: la Declaración de Doha (1991) fue una expresión de buenas intenciones para afrontar una creciente presión internacional. Mientras no se deroguen las normas que establecen la patentabilidad de los productos farmacéuticos, seguiremos sin tratar un problema grave que se pone de manifiesto en momentos de pandemia mundial como la que hoy vivimos.

2. Se denomina patente de invención a «un título otorgado por una autoridad administrativa que habilita a ejercer los derechos de exclusiva derivados de una invención, cuya vigencia está limitada en el tiempo y es revocable por incumplimiento de las cargas impuestas por la ley» (Bergel, 2013: 145).

3. Para un análisis económico exhaustivo acerca del carácter oligopólico de las empresas farmacéuticas, la distorsión y la ineficiencia económica que producen, las barreras de entrada y el aumento de los precios de los medicamentos, véase Baker (2004 y 2018). En el texto de 2018, Baker calcula que en ese año los EE. UU. gastarían 420 billones de dólares en medicinas cuyo costo en un mercado libre sería de 105 billones de dólares, una diferencia de 213 billones anuales que significaba el 1,6 por ciento del producto interior bruto. 


\section{Sobre el acceso a los medicamentos esenciales y las patentes de invención ${ }^{4}$}

La constante batalla de las compañías farmacéuticas por lograr que los medicamentos estuvieran protegidos por patentes de invención tiene su historia. Entre las tantas rutas que se ensayaron para lograr tal nefasto maridaje, preciso es recordar las rondas del GATT (Acuerdo General sobre Tarifas y Comercio), que se extendieron desde 1986 hasta 1994 y dieron lugar a la creación de la OMC (Organización Mundial del Comercio) en Uruguay en el año 1994. Lo curioso es que el objetivo de tales rondas no era discutir las patentes de invención, aunque sí un tema anejo, como lo fue la liberación del comercio internacional ('t Hoen, 2002).

El acuerdo finalmente alcanzado incorporó como documentos anexos a un conjunto de tratados relativos al comercio de bienes y servicios, entre ellos el acuerdo de los ADPIC de 1994 (Acuerdo sobre los aspectos de propiedad intelectual relacionados con el comercio. TRIPS en inglés $)^{5}$. Mediante tal convenio se introdujeron, a nivel internacional, las líneas rectoras de la propiedad intelectual que hoy nos rigen, o nos dominan.

$\mathrm{Ni}$ duda cabe que la redacción final del acuerdo refleja los intereses de un grupo de empresas globales que pugnaron por ampliar su control sobre la propiedad intelectual, y contaron con el principal apoyo del gobierno de los EE. UU., de Japón y de algunos países europeos (Abbott, 2005). El objetivo era diseñar las normas del derecho internacional público en su propio beneficio (Sell, 2008: 169).

Por otro lado, el acuerdo dejó abierta la posibilidad de extender las ventajas logradas inicialmente, que luego se ampliaron mediante un diseño de nuevos acuerdos, los llamados ADPIC-plus, que, entre otras cosas, lograron extender los periodos de protección de las patentes, las disposiciones sobre la exclusividad de los datos y el alcance de los requisitos relativos a la materia patentable. Así fue que el sector químico farmacéutico pasó a ser el que ahora mismo goza de una protección patentaria privilegiada (Roffe, 2008: 335).

En paralelo ocurrieron varias cosas, entre ellas que los ADPIC convirtieron a la Organización Mundial del Comercio en la principal recaudadora de las rentas que procedían de la propiedad intelectual, y lo hizo en nombre de las corporaciones multinacionales, lo cual muestra que finalmente resultó capturada por tales corporaciones (Comisión sobre Derechos de Propiedad Intelectual, 2002: 385). Y lo cierto es que el acuerdo de los ADPIC no fue negociado por los miembros del GATT, fue el resultado de una imposición por parte de las multinacionales con el apoyo del gobierno de los EE. UU. y de

4. Hay una extensa discusión, en la que no podemos entrar por razones de espacio, sobre qué debería considerarse un medicamento esencial. Los criterios de inclusión que utiliza la OMS son los de prevalencia, eficacia, costo y costo-eficacia (Organización Mundial de la Salud, 2019).

5. El Acuerdo de los ADPIC es el anexo 1c del Acuerdo de Marrakech, firmado el 15 de abril de 1994. OMC, anexo 1C, Acuerdo sobre los aspectos de los derechos de propiedad intelectual relacionados con el comercio. 
otros miembros. El mecanismo básico del sistema de patentes fue concebido y configurado en una declaración conjunta presentada a la secretaría del GATT en junio de 1998 por parte del Comité de Propiedad Industrial de los EE. UU., integrado entre otras por las empresas Dupont, General Electric, General Motors, Hewlett Packard, IBM, Merck, Monsanto, Pfizer, Johnson \& Johnson (Shiva, 2003: 64).

En referencia a los medicamentos, el acuerdo de los ADPIC establece lo siguiente:

1. La patentabilidad para todas las invenciones - de productos o de procedimientos - en todos los campos de la tecnología (incluyendo, naturalmente, a la farmacéutica) (art. 27).

2. El detalle del contenido de los derechos que confiere a su titular (art. 28).

3. El establecimiento del plazo de vigencia de las patentes (art. 20).

4. El establecimiento de normas jurídicas para sancionar las infracciones al régimen de patentes (art. 41.1).

Y si bien el artículo 8, en el que se exponen los principios que gobiernan al acuerdo, dice que: «al formular o modificar las leyes y reglamentos nacionales los miembros podrán adoptar las medidas necesarias para proteger la salud pública», lo cierto es que de inmediato aclara "siempre que las medidas sean compatibles con lo dispuesto en el presente Acuerdo». Ese "siempre que» es una muestra del triunfo de los intereses de la industria por encima de la salud pública y del derecho de acceso a los medicamentos. La pandemia ha dejado en claro muchas cosas, entre ellas el absurdo de una salud pública mundial sujeta a los caprichos de las grandes corporaciones farmacéuticas (Baker, 2020).

Esta historia, como muchas otras, tiene su lado siniestro para los estados más pobres, puesto que numerosos países que en ese momento no admitían la patentabilidad de los productos farmacéuticos invocando a la defensa de la salud pública, fueron obligados a hacerlo bajo la amenaza de quedar excluidos de la OMC (Ravvin, 2008). La mayoría de los países del norte, en cambio, ya habían alcanzado los niveles de protección en materia de patentes previstos en el acuerdo (Roffe, 2008: 335; Shiva, 2003: 64).

\section{Doha y las licencias obligatorias}

Durante un tiempo, con algunos altibajos y fuertes protestas por parte de activistas de organizaciones no gubernamentales, el sistema de propiedad intelectual generado por los ADPIC parecía reinar sin grandes problemas, pero la epidemia del sida — que afectó a diversos países y en forma alarmante a Sudáfrica- mostró de manera dramática cuáles eran los efectos concretos de tales acuerdos para la salud y la vida de las personas en los países pobres.

El presidente Mandela tomó la decisión de desconocer el Acuerdo mediante la denominada Ley de enmienda sudafricana de control de medicamentos 
y sustancias relacionadas, que abrió la importación de medicamentos antirretrovirales a un precio significativamente inferior a los fijados por las empresas multinacionales dueñas de las patentes ${ }^{6}$. Este hecho dramático fue lo que permitió controlar la epidemia, y la reacción de la industria farmacéutica fue inmediata. La Asociación de Fabricantes de Productos Farmacéuticos de Sudáfrica -integrada por 39 empresas multinacionales - promovió un proceso judicial por violación del Convenio de los ADPIC, proceso que incluyó a los Estados Unidos y a países europeos.

Para tener una idea de la dimensión del problema debatido, cabe recordar que en ese momento el costo anual mínimo de un tratamiento con antirretroviral excedía con creces el gasto anual per cápita en salud de la mayoría de los países en desarrollo (Comisión sobre Derechos de Propiedad Intelectual, 2002: 102). El litigio tuvo una amplia repercusión internacional y motivó una corriente crítica de los procedimientos empleados por las multinacionales, lo que llevó a las empresas actoras a desistir del pleito al cabo de tres años. Este proceso fue importante, entre otras cosas porque demostró la necesidad de que existiera el activismo internacional para discutir el acuerdo de los ADPIC (Bermudez, 2006: 58). La presión de los distintos países logró que la Organización Mundial del Comercio aprobara la denominada «Declaración de Doha relativa al acuerdo sobre los ADPIC y la salud pública».

No cabe duda de que existe un grupo de reconocidos especialistas en propiedad intelectual que otorgan a la Declaración de Doha un alcance operativo amplio. Tal es el caso de Carlos Correa, quien le atribuye el «haber sentado las bases para la diferenciación de las normativas de propiedad intelectual cuando es necesario para proteger la salud pública» (Correa, 2002). Algo similar ha escrito Seuba Hernández, para quien el valor de Doha fue demostrar que los estados tienen el derecho a interpretar el acuerdo de forma que permita proteger la salud pública y utilizar en toda su extensión las normas que contribuyan a tal fin (Seuba Hernández, 2008: 415). Y también Germán Velásquez entendió que la Declaración de Doha había puesto en evidencia que si las medicinas se consideran una simple mercancía, la salud nunca será otra cosa que una extensión del mercado, y las curas y los tratamientos solo estarán al alcance de los que disponen de suficiente poder adquisitivo (Velásquez, 2008: 27).

Los autores del presente trabajo no coincidimos con tales supuestos méritos de la Declaración de Doha en relación con la salud pública mundial. Creemos que a pesar del tono altisonante en el que fue redactada y del triunfo de una legítima lucha política momentánea, se trata de un documento estéril y poco operativo para los fines perseguidos. Nuestros argumentos, dichos muy brevemente por razones de espacio, son los siguientes:

6. Todo ello fue posible por la unión de fuerzas entre el Grupo de África y una red de países subdesarrollados, entre ellos Brasil e India, que tuvieron el apoyo de algunas ONG para lograr una gran difusión de sus demandas también en los países del Norte. La situación se polarizó de tal manera que estar a favor de la industria farmacéutica significaba, en ese momento, estar en contra del acceso universal a los medicamentos esenciales (Drahos, 2007). 
1. El artículo 4 del Documento de Doha resume los alcances de la declaración y dice: «convenimos en que el acuerdo no impide ni deberá impedir que los miembros adopten medidas para proteger la salud pública». Curiosa y ambigua formulación, como entiende Jean Claude Fritz, pues «pasamos de una obligación a una no prohibición tímida a todas luces». El «no deberá impedir», en efecto, implica ciertamente no impedir, pero también no actuar para impedirlo. A juicio de Fritz, que compartimos, la razón de tal tímida prohibición descansa en que en el marco de la OMC no son los estados entre sí los que se obligan y negocian, sino los estados y los operadores económicos de los otros estados (Fritz, 2004: 457). Es por eso que afirmamos que, en el Marco de los acuerdos multilaterales de la OMC sobre las patentes de medicamentos, las grandes empresas farmacéuticas tienen mayor poder que los estados para definir las políticas de salud pública.

2. Hemos dicho antes que no es infrecuente encontrar argumentos para sostener que las licencias obligatorias son un instrumento promisorio, una concesión por parte de la OMC para garantizar la salud pública de los países más pobres, a pesar de que el acuerdo sobre los ADPIC no menciona de manera explícita al concepto generalmente aceptado de licencias «no voluntarias» u «obligatorias» (Correa et al., 2013: 89). Sin embargo, del análisis del articulado no se sigue tal optimismo. Por ejemplo, el artículo 31 de la Declaración: "Otros usos sin autorización del titular del derecho» -el único artículo que hace referencia implícita a las licencias obligatorias-, tales licencias están sujetas a nueve condiciones, con varias aclaraciones para cada una de ellas, que en la práctica son inaplicables (Banta, 2001; Organización Mundial del Comercio, 2001). Entre tales condiciones figuran, por ejemplo, que el titular de la licencia obligatoria debería tener los conocimientos necesarios para llevar a cabo una ingeniería inversa para poder fabricar el medicamento sin la cooperación del titular de la patente ${ }^{7}$. Pero, además, que el titular solicitante debería contar con un mercado lo suficientemente amplio para justificar el costo de la inversión y la fabricación, así como pagar una remuneración adecuada al titular de la patente. Obviamente, no deberíamos descontar tampoco las dilaciones burocráticas ni jurídicas que interfieren en asuntos de salud pública en la urgencia. Con una capacidad de fabricación propia limitada no existe país subdesarrollado que sea capaz de cumplir con estas disposiciones. Irónicamente, los países desarrollados son los que han usado el sistema de licencias obligatorias en su beneficio (Comisión sobre Derechos de Propiedad Intelectual, 2002: 142).

3. Admitamos que la única disposición que podría entenderse como un cuestionamiento parcial al acuerdo sobre los ADPIC es la que encomienda al

7. Solo un pequeño grupo de los países en vías de desarrollo posee la capacidad de realizar ingeniería reserva a gran escala. Un estudio del año 1992 mostró que únicamente cinco de ellos tienen suficiente capacidad para innovar en el sector farmacéutico (definida como la capacidad de producir nuevas drogas mediante un proceso de ingeniería reversa). Esos países eran Argentina, China, India, Corea y México (Drahos, 2007: 17). 
Consejo General de la OMC que encuentre una pronta solución a las dificultades de los países que no poseen capacidad para hacer uso de las licencias obligatorias (art. 6). Y si bien el Consejo adoptó la decisión en agosto de 2003 e impulsó una enmienda del acuerdo de los ADPIC, se trata de un texto sumamente amplio y poco operativo, que exige una «remuneración adecuada» al titular de la patente y que fuera duramente cuestionado, por cuanto los procedimientos de implementación resultan lentos, burocráticos y acrecientan el precio de los medicamentos (Bermudez, 2006: 58). Las restricciones impuestas reducen la posibilidad de que dichas licencias obligatorias puedan servir como un instrumento efectivo de negociación para los países subdesarrollados.

\section{A modo de conclusión}

En un artículo anterior, los autores de este trabajo sostuvimos que «una eventual vacuna para la COVID-19 debería ser considerada como un bien público mundial» (Bertomeu y Bergel, 2020). Para lograrlo sería necesario derogar las normas que permitieron patentar los productos farmacéuticos.

La Declaración de Doha relativa al acuerdo sobre los ADPIC y la salud pública de noviembre de 2001 no es la salida para la crisis sanitaria mundial, sino una declaración con innumerables requisitos casi imposibles de ejecutar en la práctica. Doha no obliga a impedir daños a la salud pública por el aumento del precio de los medicamentos, pero además esas «no prohibiciones tímidas» de las que habla Fritz están acompañadas por una condición que disipa cualquier duda sobre sus alcances reales, porque el artículo 3 reafirma que «reconoce que la protección de la propiedad intelectual es importante para el desarrollo de nuevos medicamentos", aunque también admitan que les preocupan sus efectos sobre los precios.

En este sentido, nuestra posición es cercana a la expresada por Seuba Hernández (2014), cuando caracterizó la compleja relación entre la propiedad intelectual y los derechos humanos. Frecuentemente los documentos internacionales utilizan términos vagos y casi siempre condicionados de manera muy general por las cláusulas de los ADIPC, y la redacción de algunos artículos recogidos en tratados internacionales es ambigua y muestra un claro desequilibrio entre los derechos de los titulares y los derechos de terceros, bien sean competidores o usuarios de los productos protegidos. De ahí concluye que la redacción confusa del articulado refleja una «ambigüedad constructiva» que tiene por objeto facilitar la conclusión de un tratado, aunque existan diferentes puntos de vista sobre el significado y el alcance de los artículos (Seuba Hernández, 2014: 83).

Doha manifiesta esa «ambigüedad constructiva» en su articulado, y muy especialmente en el artículo 31, que, entre otras cosas, evita hablar de licencias obligatorias y eufemísticamente hace referencia a "otros usos no autorizados por el titular de la patente». Mientras no se deroguen las normas que establecen la patentabilidad de los productos farmacéuticos seguiremos sin afrontar 
un problema grave que se pone de manifiesto en momentos de pandemia mundial y es probable que luego se olvide. Doha no ha demostrado «no haber impedido" a los estados el desarrollo de una industria farmacéutica acorde con las necesidades de salud pública, sino todo lo contrario.

\section{Referencias bibliográficas}

Аввотт, Frederic (2005). «The Trips Agreement, Acces to Medicines and the WTO Doha Ministerial Conference». The Journal of World Intelectual Property (1 de noviembre), 15-52.

BaKer, Dean (2004). Financing Drug Research: What Are the Issues? Washington: Center for Economics and Policy Research. Recuperado el 20 de junio de 2020, de <https://www.cepr.net/documents/publications/intellectual_ property_2004_09.htm>.

- (2018). «Is intelectual Property the root of all evil?: Patents, Copyrights, and Inequality». The Great Polarization: Economics, Institutions and Policies in the Age of Inequality conference. University of Utah. Department of Economics, 27-29 de septiembre.

- (2020). «Patents Are Slowing the Development of a Coronavirus Vaccine». Environment \& Health (2 de marzo). Recuperado el 3 de marzo de 2020, de $<$ https://truthout.org/articles/patents-are-slowing-the-developmentof-a-coronavirus-vaccine/ $>$.

Banta, David (2001). «Public Health Triumphs at WTO Conference». JAMA, 286, 2655-2665.

Bergel, Salvador (2013). «Las patentes de invención». En: Correa, Carlos; Bergel, Salvador y Korks, Jorge. Régimen legal de las patentes de invención. Ciudad Autónoma de Buenos Aires: La Ley.

Bermudez, Jorge y Oliveira, María Auxiliadora (2006). La propiedad intelectual en el contexto del acuerdo de la OMC sobre los ADPIC. Río de Janeiro: Fio Cruz.

Bertomeu, Maria Julia y Bergel, Salvador (2020). «La eventual vacuna para Covid-19 debería ser considerada como un bien público mundial: Sobre el Proyecto de Resolución de la 73 Asamblea Mundial de la Salud». Sin Permiso, 16 (19 de mayo). Recuperado el 27 de mayo de 2020, de <https:// www.sinpermiso.info/textos/la-eventual-vacuna-para-covid-19-deberiaser-considerada-como-un-bien-publico-mundial-sobre-el>.

Comisión sobre Derechos de Propiedad Intelectual (2002). Integrando los derechos de propiedad intelectual y la politica del desarrollo. Londres.

Correa, Carlos (2002). Repercusiones de la Declaración de Doha relativa al acuerdo sobre los ADPIC y la salud pública. Ciudad Autónoma de Buenos Aires: Universidad de Buenos Aires. Recuperado el 5 de mayo de 2020, de <https://apps.who.int/iris/bitstream/handle/10665/68356/WHO_EDM_ PAR_2002.3_spa.pdf?sequence $=1$ \&isAllowed $=y>$.

Correa, Carlos; Bergel, Salvador y Korks, Jorge (2013). Régimen legal de las patentes de invención. Ciudad Autónoma de Buenos Aires: La Ley. 
Drahos, Peter (2007). «Four lessons for developing countries from the trade negotiations over Access to medicines». Liverpool Law Review, 28, 11-39. <https://doi.org/10.1007/s10991-007-9014-5>

FrITZ, Jean Claude (2004). «A manera de ilustración: La Declaración de Doha, ¿viraje o no?». En: SÁNCHez Rubio et al. (2004). Nuevos colonialismos del capital. Barcelona: Icaria.

Organización Mundial de la SAlud (OMS) (2019). La OMS actualiza sus orientaciones mundiales sobre medicamentos y pruebas diagnósticas para abordar problemas de salud especificos, priorizar los tratamientos más eficaces y mejorar el acceso a terapias asequibles (9 de julio). Recuperado el 20 de junio de 2020, de <https://www.who.int/es/news-room/detail/09-07-2019who-updates-global-guidance-on-medicines-and-diagnostic-tests-toaddress-health-challenges-prioritize-highly-effective-therapeutics-andimprove-affordable-access $>$.

Organización Mundial del Comercio (OMC) (1994). Anexo 1C: Acuerdo sobre los derechos de propiedad intelectual relacionados con el comercio. Recuperado el 20 de junio de 2020, de <https://www.wto.org/spanish/docs_s/ legal_s/27-trips.pdf>.

- (2001). Doha Ministerial Declaration on the TRIPS Agreement and Public Health. Recuperado el 3 de mayo de 2020, de <https://www.wto.org/english/ thewto_e/minist_e/min01_e/mindecl_trips_e.htm>.

Ravvin, Michael (2008). «Incentivizing Access and Innovation for Essential Medicines: A Survey of the problema and Proposed Solutions». Public Health Ethics, 1 (2), 110-123.

Roffe, Pedro (2008). "La cuestión de la "materia existente" y los productos farmacéuticos». En: Seuba Hernández, Xavier. Salud pública y productos farmacéuticos. Barcelona: Bosch, 337-387.

Sell, Susan (2008). «Acuerdos de libre comercio con disposiciones ADPIC plus». En: Seuba Hernández, Xavier. Salud pública y productos farmacéuticos. Barcelona: Bosch, 171-215.

Seuba Hernández, Xavier (2008). Salud pública y productos farmacéuticos. Barcelona: Bosch.

- (2014). «Derechos fundamentales y observancia de la propiedad intelectual». Revista del Estudio del Derecho y Ciencia Política, 17, 78-96.

Shiva, Vandana (2003). ¿Proteger o expoliar?: Los derechos de propiedad intelectual. Barcelona: Intercom.

't Hoen, Ellen (2002). «TRIPS, Pharmaceutical Patents, and Access to Essential Medicines: A Long Way From Seattle to Doha». Chicago Journal of International Law, 3 (1).

VelásQuez, Germán (2008). «El acceso a los medicamentos en perspectiva global: Retos, respuestas y derechos». En: Seuba Hernández, Xavier. Salud pública y productos farmacéuticos. Barcelona: Bosch, 17-41. 
Salvador Bergel es profesor titular de la Cátedra de Bioética de la Universidad de Buenos Aires.

Salvador Bergel is Full Professor of the Chair of Bioethics at the University of Buenos Aires.

María Julia Bertomeu es miembro del Consejo Nacional de Investigaciones Científicas y Técnicas. CIF-INEO. Sus temas de investigación son 1) Kant, en la tradición política republicana clásica, con especial atención a los conceptos de propiedad y libertad, y 2) usos del concepto de solidaridad en el discurso bioético.

María Julia Bertomeu is member of the CIF-INEO National Council for Scientific and Technical Research. Her research topics include Kant in the classic republican political tradition, focusing on the concepts of property and freedom, and uses of the concept of solidarity in bioethical discourse. 